\title{
NOTES ON DOUBLE INEQUALITIES OF MATHIEU'S SERIES
}

FENG QI, CHAO-PING CHEN, AND BAI-NI GUO

Received 7 January 2005 and in revised form 5 July 2005

Using the integral expression of Mathieu's series and some integral and analytic inequalities involving periodic functions and the generating function of Bernoulli numbers, we present several new inequalities and estimates for Mathieu's series and generalize Mathieu's series. Two open problems are proposed.

\section{Introduction}

In 1890, Mathieu defined $S(r)$ in [10] as

$$
S(r)=\sum_{n=1}^{\infty} \frac{2 n}{\left(n^{2}+r^{2}\right)^{2}}, \quad r>0,
$$

and conjectured that $S(r)<1 / r^{2}$. We call formula (1.1) Mathieu's series.

This conjecture was proved in 1952 by Berg in [2]. Since then, various papers appeared providing interesting new inequalities involving $S(r)$. Please refer to references listed in this paper.

In [9], Makai proved

$$
\frac{1}{r^{2}+1 / 2}<S(r)<\frac{1}{r^{2}}
$$

Alzer et al. in [1] obtained

$$
\frac{1}{x^{2}+1 /(2 \zeta(3))}<S(x)<\frac{1}{x^{2}+1 / 6}
$$

where $\zeta$ denotes the zeta function. The inequalities in (1.3) are sharp: the constants $1 /(2 \zeta(3))$ and $1 / 6$ are the best possibe.

The integral form of Mathieu's series (1.1) was given in $[3,4]$ by

$$
S(r)=\frac{1}{r} \int_{0}^{\infty} \frac{x}{e^{x}-1} \sin (r x) d x .
$$


2548 Notes on double inequalities of Mathieu's series

In this paper, using the integral expression (1.4) of Mathieu's series and certain inequalities involving periodic functions and the generating function of Bernoulli numbers, some new inequalities and estimates for Mathieu's series are established and Mathieu's series is generalized. At the end, two open problems are proposed.

\section{General results}

In this section, we will establish several general theorems and inequalities involving periodic functions and then obtain some general inequalities for Mathieu's series.

Lemma 2.1. Let $\psi(x)$ be an integrable function satisfying $\psi(x)=-\psi(x+T)$, where $T$ is a given positive number, and $\psi(x) \geq 0$ for $x \in[0, T]$, let $f(x)$ and $g(x)$ be two integrable functions on $[0,2 T]$ such that

$$
f(x)-g(x) \geq f(x+T)-g(x+T)
$$

on $[0, T]$. Then

$$
\int_{0}^{2 T} \psi(x) f(x) d x \geq \int_{0}^{2 T} \psi(x) g(x) d x .
$$

Proof. By easy computation, it is deduced that

$$
\begin{aligned}
\int_{0}^{2 T} \psi(x)[f(x)-g(x)] d x \\
\quad=\int_{0}^{T} \psi(x)[f(x)-g(x)] d x+\int_{T}^{2 T} \psi(x)[f(x)-g(x)] d x \\
\quad=\int_{0}^{T} \psi(x)[f(x)-g(x)] d x+\int_{0}^{T} \psi(x+T)[f(x+T)-g(x+T)] d x \\
\quad=\int_{0}^{T} \psi(x)\{[f(x)-g(x)]-[f(x+T)-g(x+T)]\} d x \\
\quad \geq 0 .
\end{aligned}
$$

The proof is complete.

Corollary 2.2. Let $\psi(x) \neq 0$ be an integrable periodic function with period $2 T>0$ satisfying $\psi(x)=-\psi(x+T)$ and $\psi(x) \geq 0$ for $x \in[0, T]$. If $f(x)$ is an integrable function such that $f(x) \geq f(x+T)$ on $[0, T]$, then

$$
\int_{0}^{2 T} \psi(x) f(x) d x \geq 0 .
$$

Corollary 2.3. Let $f(x)$ be an integrable function such that $f(x) \geq f(x+\pi)$ on $[0, \pi]$, then

$$
\int_{0}^{2 \pi} f(x) \sin x d x \geq 0
$$

As a direct consequence of Lemma 2.1, we have the following theorem. 
Theorem 2.4. Let $\Phi_{1}$ and $\Phi_{2}$ be two integrable functions such that $x /\left(e^{x}-1\right)-\Phi_{1}(x)$ and $\Phi_{2}(x)-x /\left(e^{x}-1\right)$ are both increasing. Then, for any positive number $r$,

$$
\frac{1}{r} \int_{0}^{\infty} \Phi_{2}(x) \sin (r x) d x \leq \sum_{n=1}^{\infty} \frac{2 n}{\left(n^{2}+r^{2}\right)^{2}} \leq \frac{1}{r} \int_{0}^{\infty} \Phi_{1}(x) \sin (r x) d x .
$$

Proof. The function $\psi(x)=\sin (r x)$ has a period $2 \pi / r$, and $\psi(x)=-\psi(x+\pi / r)$.

Since $f(x)=x /\left(e^{x}-1\right)-\Phi_{1}(x)$ is increasing, for any $\alpha>0$, we have $f(x+\alpha) \geq f(x)$. Therefore, from Corollary 2.2, we obtain

$$
\begin{gathered}
\int_{2 k \pi / r}^{2(k+1) \pi / r}\left[\frac{x}{e^{x}-1}-\Phi_{1}(x)\right] \sin (r x) d x \leq 0, \\
\int_{2 k \pi / r}^{2(k+1) \pi / r} \frac{x}{e^{x}-1} \sin (r x) d x \leq \int_{2 k \pi / r}^{2(k+1) \pi / r} \Phi_{1}(x) \sin (r x) d x .
\end{gathered}
$$

Then, from formula (1.4), we have

$$
\begin{aligned}
S(r) & =\frac{1}{r} \sum_{k=0}^{\infty} \int_{2 k \pi / r}^{2(k+1) \pi / r} \frac{x}{e^{x}-1} \sin (r x) d x \\
& \leq \frac{1}{r} \sum_{k=0}^{\infty} \int_{2 k \pi / r}^{2(k+1) \pi / r} \Phi_{1}(x) \sin (r x) d x \\
& =\frac{1}{r} \int_{0}^{\infty} \Phi_{1}(x) \sin (r x) d x .
\end{aligned}
$$

The right-hand side of inequality (2.6) follows.

Similar arguments yield the left-hand side of inequality (2.6).

\section{The first concrete result}

Using Theorem 2.4 obtained in the previous section, now we will give the first concrete estimate for Mathieu's series by monotonicity of difference between a function related to the exponential function and the generating function $x /\left(e^{x}-1\right)$ of Bernoulli numbers.

Proposition 3.1. The function

$$
g(x)=\frac{x}{e^{x}-1}-\frac{x^{2}}{e^{3 x}-e^{x}}
$$

is decreasing with $x>0$.

Proof. The proof follows from elementary analysis and standard argument.

Theorem 3.2. For any positive number $r>0$,

$$
\sum_{n=1}^{\infty} \frac{2 n}{\left(n^{2}+r^{2}\right)^{2}} \geq \frac{2\left(r^{2}-3\right)}{\left(1+r^{2}\right)^{3}}+\frac{\pi^{3}}{8 r} \operatorname{sech}^{2}\left(\frac{\pi r}{2}\right) \tanh \left(\frac{\pi r}{2}\right) .
$$


2550 Notes on double inequalities of Mathieu's series

Proof. In [15, page 356], the following formula is given:

$$
\int_{0}^{\infty} \frac{x^{2 m} \sin (a x)}{e^{(2 n+1) \alpha x}-e^{(2 n-1) \alpha x}} d x=(-1)^{m} \frac{\partial^{2 m}}{\partial a^{2 m}}\left[\frac{\pi}{4 \alpha} \tanh \frac{a \pi}{2 \alpha}-\sum_{\nu=1}^{n} \frac{a}{a^{2}+(2 v-1)^{2} \alpha^{2}}\right]
$$

where $\alpha>0$ and $m, n=0,1,2, \ldots$ If $n=0$ in formula (3.3), then the summation terms are omitted.

Therefore, we have

$$
\begin{aligned}
\int_{0}^{\infty} \frac{x^{2} \sin (r x)}{e^{3 x}-e^{x}} d x & =-\frac{\partial^{2}}{\partial r^{2}}\left[\frac{\pi}{4} \tanh \frac{\pi r}{2}-\frac{r}{r^{2}+1}\right] \\
& =\frac{1}{8\left(1+r^{2}\right)^{3}}\left[16 r\left(r^{2}-3\right)+\pi^{3}\left(r^{2}+1\right)^{3} \operatorname{sech}^{2}\left(\frac{\pi r}{2}\right) \tanh \left(\frac{\pi r}{2}\right)\right]
\end{aligned}
$$

From Theorem 2.4 and Proposition 3.1, inequality (3.2) follows.

\section{The second concrete result}

In this section, by an inequality relating to the generating function $x /\left(e^{x}-1\right)$ of Bernoulli numbers, using the periodicity of the sine function, and reducing the integral expression (1.4) of Mathieu's series to another series, we will formulate another meaningful estimate to Mathieu's series.

Proposition 4.1. For $x>0$,

$$
\frac{1}{e^{x}}<\frac{x}{e^{x}-1}<\frac{1}{e^{x / 2}}
$$

Proof. This follows from standard argument.

Theorem 4.2. For any positive number $r>0$,

$$
\frac{4\left(1+r^{2}\right)\left(e^{-\pi / r}+e^{-\pi /(2 r)}\right)-4 r^{2}-1}{\left(e^{-\pi / r}-1\right)\left(1+r^{2}\right)\left(1+4 r^{2}\right)} \leq S(r) \leq \frac{\left(1+4 r^{2}\right)\left(e^{-\pi / r}-e^{-\pi /(2 r)}\right)-4\left(1+r^{2}\right)}{\left(e^{-\pi / r}-1\right)\left(1+r^{2}\right)\left(1+4 r^{2}\right)}
$$

Proof. For $r>0$, using (1.4), by direct calculation, we have

$$
S(r)=\frac{1}{r} \sum_{k=0}^{\infty}\left[\int_{2 k \pi / r}^{(2 k+1) \pi / r} \frac{x \sin (r x)}{e^{x}-1} d x+\int_{(2 k+1) \pi / r}^{(2 k+2) \pi / r} \frac{x \sin (r x)}{e^{x}-1} d x\right]
$$


The inequality (4.1) gives us

$$
\begin{aligned}
\frac{r\left(1+e^{-\pi / r}\right)}{\left(1+r^{2}\right)\left(1-e^{-2 \pi / r}\right)} & =\sum_{k=0}^{\infty} \int_{2 k \pi / r}^{(2 k+1) \pi / r} \frac{\sin (r x)}{e^{x}} d x \leq \sum_{k=0}^{\infty} \int_{2 k \pi / r}^{(2 k+1) \pi / r} \frac{x \sin (r x)}{e^{x}-1} d x \\
& \leq \sum_{k=0}^{\infty} \int_{2 k \pi / r}^{(2 k+1) \pi / r} \frac{\sin (r x)}{e^{x / 2}} d x=\frac{4 r\left(1+e^{-\pi /(2 r)}\right)}{\left(1+4 r^{2}\right)\left(1-e^{-\pi / r}\right)}, \\
-\frac{4 r\left(e^{-\pi / r}+e^{-\pi /(2 r)}\right)}{\left(1+4 r^{2}\right)\left(1-e^{-\pi / r}\right)} & =\sum_{k=0}^{\infty} \int_{(2 k+1) \pi / r}^{2(k+1) \pi / r} \frac{\sin (r x)}{e^{x / 2}} d x \leq \sum_{k=0}^{\infty} \int_{(2 k+1) \pi / r}^{2(k+1) \pi / r} \frac{x \sin (r x)}{e^{x}-1} d x \\
& \leq \sum_{k=0}^{\infty} \int_{(2 k+1) \pi / r}^{2(k+1) \pi / r} \frac{\sin (r x)}{e^{x}} d x=-\frac{r\left(e^{-2 \pi / r}+e^{-\pi / r}\right)}{\left(1+r^{2}\right)\left(1-e^{-2 \pi / r}\right)} .
\end{aligned}
$$

Substituting (4.4) into (4.3) yields

$$
\begin{aligned}
& \frac{4 e^{-\pi / r}+4 r^{2} e^{-\pi / r}+4 e^{-\pi /(2 r)}+4 r^{2} e^{-\pi /(2 r)}-4 r^{2}-1}{\left(e^{-\pi / r}-1\right)\left(1+r^{2}\right)\left(1+4 r^{2}\right)} \\
& =\frac{1+e^{-\pi / r}}{\left(1+r^{2}\right)\left(1-e^{-2 \pi / r}\right)}-\frac{4\left(e^{-\pi / r}+e^{-\pi /(2 r)}\right)}{\left(1+4 r^{2}\right)\left(1-e^{-\pi / r}\right)} \\
& \leq S(r) \\
& \leq \frac{4\left(1+e^{-\pi /(2 r)}\right)}{\left(1+4 r^{2}\right)\left(1-e^{-\pi / r}\right)}-\frac{e^{-2 \pi / r}+e^{-\pi / r}}{\left(1+r^{2}\right)\left(1-e^{-2 \pi / r}\right)} \\
& =\frac{e^{-\pi / r}+4 r^{2} e^{-\pi / r}-4 e^{-\pi /(2 r)}-4 r^{2} e^{-\pi /(2 r)}-4-4 r^{2}}{\left(e^{-\pi / r}-1\right)\left(1+r^{2}\right)\left(1+4 r^{2}\right)} \text {. }
\end{aligned}
$$

The proof is complete.

Remark 4.3. When $0<r<0.83273 \ldots$, the upper bound in (4.2) is better than that in (1.3). In fact, straightforward computation yields

$$
\lim _{r \rightarrow 0} \frac{\left(1+4 r^{2}\right)\left(e^{-\pi / r}-e^{-\pi /(2 r)}\right)-4\left(1+r^{2}\right)}{\left(e^{-\pi / r}-1\right)\left(1+r^{2}\right)\left(1+4 r^{2}\right)}=4<6=\lim _{r \rightarrow 0} \frac{1}{r^{2}+1 / 6} .
$$

When $0<r<2.9002 \ldots$, the lower bound in (4.2) is positive, and then is useful. But, it is not better than that in (1.3).

\section{The third concrete result}

In this section, we will give another result using an approach similar to that in the previous section.

Theorem 5.1. For any positive number $r>0$,

$$
\sum_{n=1}^{\infty} \frac{2 n}{\left(n^{2}+r^{2}\right)^{2}}<\frac{1}{r} \int_{0}^{\pi / r} \frac{x}{e^{x}-1} \sin (r x) d x<\frac{1+\exp (-\pi /(2 r))}{r^{2}+1 / 4} .
$$


2552 Notes on double inequalities of Mathieu's series

Proof. Straightforward computation yields

$$
\begin{aligned}
\int_{0}^{\infty} \frac{x}{e^{x}-1} \sin (r x) d x-\int_{0}^{\pi / r} \frac{x}{e^{x}-1} \sin (r x) d x \\
\quad=\sum_{k=1}^{\infty} \int_{k \pi / r}^{(k+1) \pi / r} \frac{x}{e^{x}-1} \sin (r x) d x \\
\quad=\sum_{i=1}^{\infty}\left(\int_{2 i \pi / r}^{(2 i+1) \pi / r}+\int_{(2 i-1) \pi / r}^{2 i \pi / r}\right) \frac{x}{e^{x}-1} \sin (r x) d x \\
=\sum_{i=1}^{\infty}\left(\int_{0}^{\pi}+\int_{-\pi}^{0}\right) \frac{(2 i \pi+t) / r}{\exp ((2 i \pi+t) / r)-1} \sin (2 i \pi+t) \frac{d t}{r} \\
=-\frac{1}{r} \sum_{k=1}^{\infty} \int_{0}^{\pi}\left[\frac{[(2 k-1) \pi+t] / r}{\exp ([(2 k-1) \pi+t] / r)-1}-\frac{(2 k \pi+t) / r}{\exp ((2 k \pi+t) / r)-1}\right] \sin t d t .
\end{aligned}
$$

It is easy to see that the function $\left(e^{t}-1\right) / t$ is strictly increasing on $(0,+\infty)$, therefore, for all $t>0$, we have

$$
\frac{[(2 k-1) \pi+t] / r}{\exp ([(2 k-1) \pi+t] / r)-1}>\frac{(2 k \pi+t) / r}{\exp ((2 k \pi+t) / r)-1} .
$$

Then, from inequality (4.1), we have

$$
\int_{0}^{\infty} \frac{x}{e^{x}-1} \sin (r x) d x<\int_{0}^{\pi / r} \frac{x}{e^{x}-1} \sin (r x) d x<\int_{0}^{\pi / r} e^{-x / 2} \sin (r x) d x=\frac{r(1+\exp (-\pi / 2 r))}{r^{2}+1 / 4}
$$

Inequality (5.1) follows from combining (5.4) with (1.4).

Remark 5.2. The monotonicity and convexity of the function $\left(e^{t}-1\right) / t$ can be deduced from those of the function $\left(b^{t}-a^{t}\right) / t$. For details, please refer to $[6,7,8,11,12,13,14]$.

Remark 5.3. If $r>1.57482 \ldots$, the upper bound in (5.1) is better than that in (4.2). When $r<1.574816 \ldots$, the upper bound in (5.1) is not better than that in (4.2). When $0<r<$ $0.734821 \ldots$, the upper bound in (5.1) is better than that in (1.3).

\section{Open problems}

Now we would like to propose two open problems as follows.

Open Problem 6.1. Let

$$
S(r, t, \alpha)=\sum_{n=1}^{\infty} \frac{2 n^{\alpha / 2}}{\left(n^{\alpha}+r^{2}\right)^{t+1}}
$$

for $t>0, r>0$, and $\alpha>0$. Can one obtain an integral expression of $S(r, t, \alpha)$ ? Give some sharp inequalities for the series $S(r, t, \alpha)$. 
In [15, page 356], the following formula is given

$$
\int_{0}^{\infty} \frac{x^{2 m} \sin (a x)}{e^{2 n \alpha x}-e^{(2 n-2) \alpha x}} d x=(-1)^{m} \frac{\partial^{2 m}}{\partial a^{2 m}}\left[\frac{\pi}{4 \alpha} \operatorname{coth} \frac{a \pi}{2 \alpha}-\frac{1}{2 a}-\sum_{\nu=1}^{n-1} \frac{a}{a^{2}+(2 v)^{2} \alpha^{2}}\right]
$$

where $a>0, \alpha>0, m=0,1,2, \ldots$, and $n=1,2, \ldots$ If $n=1$ in formula (6.2), then the summation terms are omitted.

Remark 6.2. One can also find the formulae (3.3) and (6.2) in other handbooks on integral formulae.

Open Problem 6.3. Find suitable ranges of numbers $\alpha, m$, and $n$ such that

$$
\begin{gathered}
\frac{x}{e^{x}-1}-\frac{x^{2 m}}{e^{(2 n+1) \alpha x}-e^{(2 n-1) \alpha x}}, \quad \alpha>0 \text { and } m, n=0,1,2, \ldots, \\
\frac{x}{e^{x}-1}-\frac{x^{2 m}}{e^{2 n \alpha x}-e^{(2 n-2) \alpha x}}, \quad \alpha>0, m=0,1,2, \ldots \text { and } n=1,2, \ldots,
\end{gathered}
$$

are monotonic in $x$.

Remark 6.4. If one can give an answer to Open Problem 6.3, then, maybe a better upper bound for Mathieu's series (1.1) could be obtained.

Remark 6.5. In [5], several inequalities of the series $S(r, t, \alpha)$ for $0<r<1$ are established and Open Problem 6.1 is solved in the following cases:

(1) $\alpha=2$ and $t$ is a natural number with $t>1$;

(2) $\alpha=2$ and $t=k-1 / 2$, where $k$ is a natural number.

\section{Acknowledgments}

The authors were supported in part by the Natural Science Foundation of China, Grant 10001016, Science Foundation for the Prominent Youth of Henan Province, Grant 0112000200, the Science Foundation of Project for Fostering Innovation Talents at Universities of Henan Province, Natural Science Foundation of Henan Province, Grant 004051800, SF for Pure Research of Natural Science of the Education Department of Henan Province (\#1999110004), Doctor Fund of Henan Polytechnic University, China. The authors would like to express heartily thanks to the anonymous referees and editors for their many helpful comments and careful editing of language expressions in this paper.

\section{References}

[1] H. Alzer, J. L. Brenner, and O. G. Ruehr, On Mathieu's inequality, J. Math. Anal. Appl. 218 (1998), no. 2, 607-610.

[2] L. Berg, Über eine Abschätzung von Mathieu, Math. Nachr. 7 (1952), 257-259 (German).

[3] Á. Elbert, Asymptotic expansion and continued fraction for Mathieu's series, Period. Math. Hungar. 13 (1982), no. 1, 1-8.

[4] O. Emersleben, Über die Reihe $\sum_{k=1}^{\infty} k /\left(k^{2}+c^{2}\right)^{2}$, Math. Ann. 125 (1952), no. 1, 165-171 (German). 
[5] I. Gavrea, Some remarks on Mathieu's series, Mathematical Analysis and Approximation Theory, Burg, Sibiu, 2002, pp. 113-117.

[6] B.-N. Guo and F. Qi, Generalization of Bernoulli polynomials, Internat. J. Math. Ed. Sci. Tech. 33 (2002), no. 3, 428-431.

[7] Q.-M. Luo, B.-N. Guo, and F. Qi, Generalizations of Bernoulli's numbers and polynomials, RGMIA Res. Rep. Coll. 5 (2002), no. 2, Art. 5, 353-359, Available online at http://rgmia.vu.edu.au/v5n2.html.

[8] Q.-M. Luo, B.-N. Guo, F. Qi, and L. Debnath, Generalizations of Bernoulli numbers and polynomials, Int. J. Math. Math. Sci. 2003 (2003), no. 59, 3769-3776.

[9] E. Makai, On the inequality of Mathieu, Publ. Math. Debrecen 5 (1957), 204-205.

[10] E. Mathieu, Traité de physique mathématique, VI-VII: Théorie de l'élasticité des corps solides, Gauthier-Villars, Paris, 1890.

[11] F. Qi, The extended mean values. Definition, properties, monotonicities, comparison, convexities, generalizations, and applications, RGMIA Res. Rep. Coll. 5 (2002), no. 1, Art. 12, 57-80, Available online at http://rgmia.vu.edu.au/v5n1.html.

[12] The extended mean values. Definition, properties, monotonicities, comparison, convexities, generalizations, and applications, Cubo Mat. Educ. 5 (2003), no. 3, 63-90.

[13] F. Qi and B.-N. Guo, Generalization of Bernoulli polynomials, RGMIA Res. Rep. Coll. 4 (2001), no. 4, Art. 10, 691-695, Available online at http://rgmia.vu.edu.au/v4n4.html.

[14] F. Qi and S.-L. Xu, The function $\left(b^{x}-a^{x}\right) / x$ : inequalities and properties, Proc. Amer. Math. Soc. 126 (1998), no. 11, 3355-3359.

[15] F.-W. Zou, Zh.-Zh. Liu, and H.-Ch. Zhou, Jîfên Biăo Hùibiān [Collection of Integral Formulas], Space Navigation Press, Beijing, 1992.

Feng Qi: Research Institute of Mathematical Inequality Theory, Henan Polytechnic University, Jiaozuo City, Henan Province 454010, China

E-mail addresses: qifeng@hpu.edu.cn; fengqi618@member.ams.org

Chao-Ping Chen: School of Mathematics and Informatics, Henan Polytechnic University, Jiaozuo City, Henan Province 454010, China

E-mail address: guobaini@hpu.edu.cn

Bai-Ni Guo: School of Mathematics and Informatics, Henan Polytechnic University, Jiaozuo City, Henan Province 454010, China

E-mail address: chenchaoping@hpu.edu.cn 


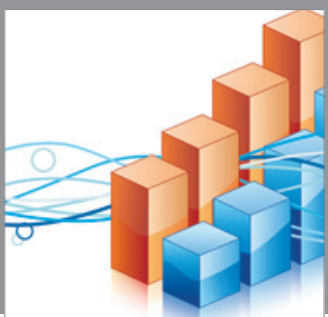

Advances in

Operations Research

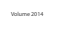

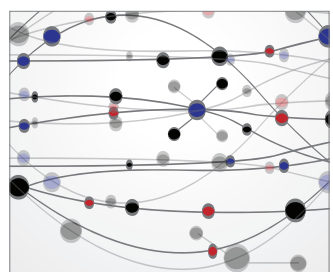

\section{The Scientific} World Journal
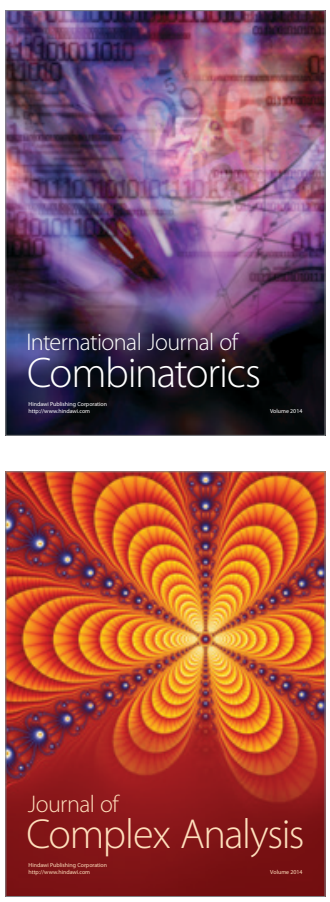

International Journal of

Mathematics and

Mathematical

Sciences
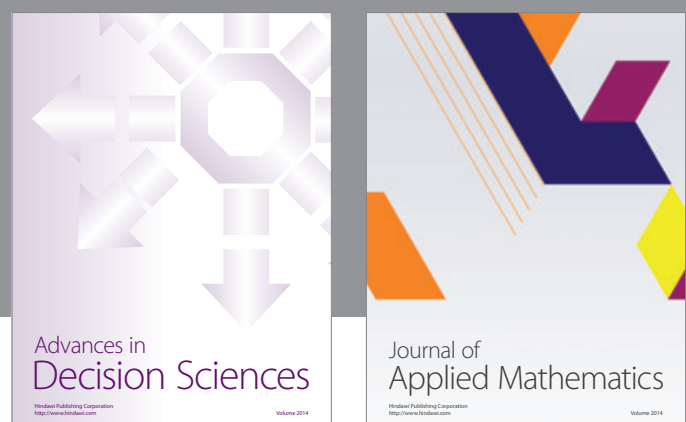

Journal of

Applied Mathematics
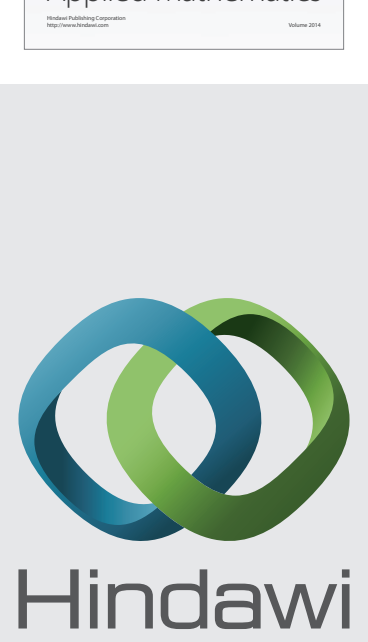

Submit your manuscripts at http://www.hindawi.com
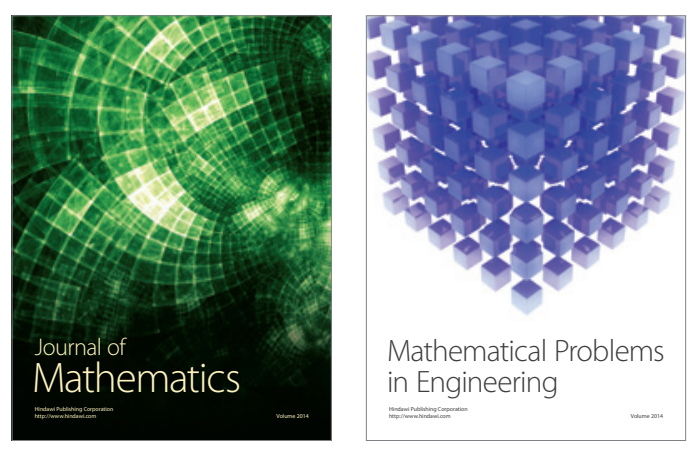

Mathematical Problems in Engineering
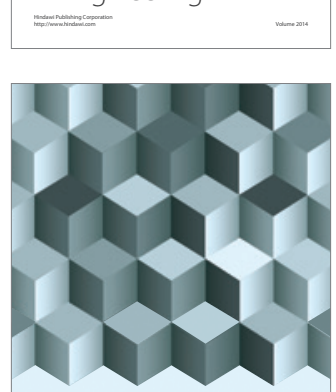

Journal of

Function Spaces
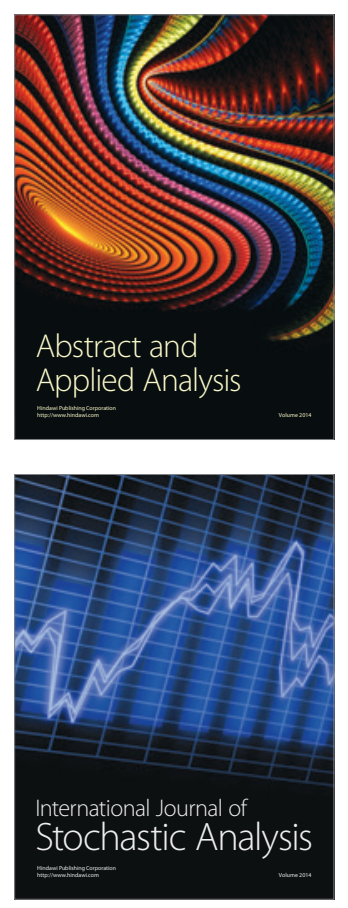

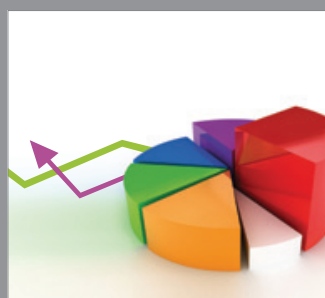

ournal of

Probability and Statistics

Promensencen
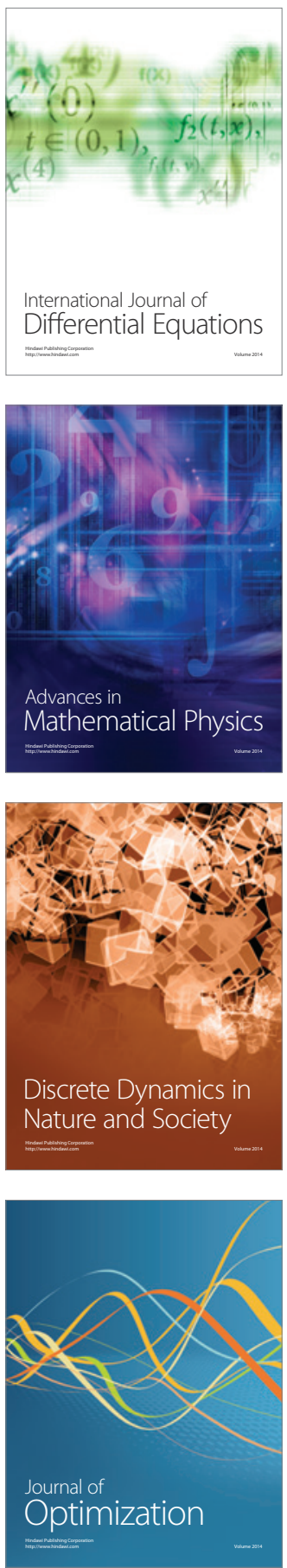